\title{
Incidence, Management and Outcome of Atonic Postpartum Haemorrhage at Tertiary Care Hospital
}

\author{
Vidyadhar Bangal ${ }^{*}$ \\ Department of Obstetrics and Gynaecology, Rural Medical College, Pravara Institute of Medical Sciences, Loni, \\ India
}

*Corresponding Author: Vidyadhar Bangal, Department of Obstetrics and Gynaecology, Rural Medical College, Pravara Institute of Medical Sciences, Loni, India, Tel: 9145154778; E-mail: vbb217@rediffmail.com

Received: 16 April 2018; Accepted: 30 April 2018; Published: 01 May 2018

\section{Abstract}

Aim: To study incidence, management and outcome of atonic postpartum haemorrhage.

Methods: Cross sectional study design. Analysis of delivery data of one year at Pravara Rural Hospital.

Results: There were 116 cases of atonic PPH. The incidence of atonic PPH was 0.88 percent. Atonic PPH was more in caesarean deliveries as compared to vaginal deliveries. It was more in emergency caesarean sections as compared to elective caesarean. There were 54 cases (46.55\%) of mild $\mathrm{PPH}$, who were successfully managed by medical methods. Uterine balloon tamponade was used in those cases who did not respond to medical management (31.89\%). Surgical intervention in the form of either B Lynch suture or stepwise devascularisation including internal iliac ligation was performed in 5 cases and obstetric hysterectomy was required in 20 cases. There were two maternal deaths among 116 cases of atonic PPH.

Conclusion: Atonic Postpartum haemorrhage is common among high risk pregnancies. Institutional deliveries will help in preventing and managing this complication in a effective manner. Facility Preparedness in all respect will prevent deaths due to atonic PPH. The incidence can be lowered by universal adoption of AMTSL. Wise and prompt decisions regarding use of different modalities or combination of modalities will save lives.

Keywords: Atonic PPH; Maternal Mortality; Obstetric Hysterectomy; Stepwise devascularisation of pelvis; Uterine balloon tamponade 


\section{Introduction}

Postpartum haemorrhage is one of the most common causes of maternal deaths throughout the world. Worldwide 5,00,000-6,00,000 maternal deaths take place annually during childbirth,of which $25 \%$ are due to postpartum hemorrhage. World Health Organization also estimated 20 million annual maternal morbidities due to obstetric haemorrhage. In developing countries, where maternal mortality rates are exponentially high, postpartum haemorrhage plays an even greater roll [1]. World Health Organization defines postpartum haemorrhage as blood loss of more than $500 \mathrm{ml}$ during the third stage of labour or during the first 24 hours after delivery [2]. It occurs in approximately 3- 4\% of vaginal deliveries [3].

Postpartum haemorrhage (PPH) continues to be the most common cause of maternal mortality in India. Approximately 20-25 percent of the maternal deaths are attributed to postpartum haemorrhage. Analysis of the deaths reveal that majority of these deaths could have been prevented by recognition of high risk factors, institutional deliveries and timely transfer of case to tertiary care hospital. Various delays are attributed to deterioration of condition of the women when they are finally admitted to higher centres.

\section{Objectives}

1. To find out the incidence of atonic postpartum haemorrhage.

2. To analyse various modalities used in the management of atonic PPH.

3. To study maternal outcome in PPH.

4. To suggest measures to reduce the incidence and improve the outcome following PPH.

\section{Material and Methods}

A retrospective record based analytical study was carried out at Pravara Rural Hospital Loni. Data related to deliveries that took place from March 2017 to February 2018 was considered for analysis. All cases of postpartum haemorrhage were included. Cases referred from outside with postpartum haemorrhage were analysed separately. Data was collected from labour room register, admission register, PPH and Maternal mortality register, Blood bank registers, operation theatre register. Data was compiled and analysed using SPSS software version 21. Data was presented in percentages.

\section{Results}

Out of 9351 deliveries,83 cases had atonic PPH during study period. The incidence of atonic PPH was 0.88 percent. There were 6339 vaginal deliveries and 3012 caesarean sections, giving incidence of $0.80 \%$ and $1.06 \%$ respectively. The incidence of atonic PPH was $0.99 \%$ and $1.10 \%$ for elective and emergency caesarean sections respectively. There were 33 referred cases of atonic PPH during study period. Out of 33 cases, 22 had vaginal delivery and 11 had undergone caesarean section in private or government hospitals. 
Out of total 73 cases of PPH, who had delivered vaginally, 22 (30.13\%) were treated by medical management, 44 (60.27\%) were treated with combined medical management and uterine balloon tamponade and 7 (9.58\%) cases required surgical intervention. Out of total 43 cases of PPH, who had caesarean section, 23 (53.48\%) were treated by medical management, 2 (4.65\%) were treated with combined medical management and uterine balloon tamponade and 18 (41.86\%) cases required surgical intervention.

Uterine balloon tamponade was successfully used in 28 cases. Haemorrhage could be stopped in all 28 cases. The balloon was kept in uterus for average of 10-14 hours. Balloon was inflated with average of $650 \mathrm{ml}$ of fluid. The balloon was retained in all cases except one. Surgical intervention in the form of either B Lynch suture or stepwise devascularisation including internal iliac ligation was performed in 5 cases and obstetric hysterectomy was required in 20 cases. Obstetric hysterectomies were performed in severe cases of atonic postpartum haemorrhage. Majority of cases had associated severe hypertension and accidental haemorrhage with features of couvelarie uterus. There were two maternal deaths due to atonic PPH and both the cases were referred from outside with irreversible shock.

\section{Discussion}

Postpartum haemorrhage is a leading cause of maternal mortality. The place of delivery and severity of haemorrhage determine the outcome. If the woman has developed PPH following delivery in a health facility, the immediate medical and surgical interventions are possible. It is not so, when woman delivers at home or in a small hospital illequiped with facilities to manage obstetric emergencies. Diagnosis of PPH and decision to transfer to hospital or tertiary care centre is very crucial. Home deliveries and deliveries in small facilities have negative influence on the outcome. Crucial time is lost in transfer of patient to higher centres. At times patients are transferred as per transfer protocols of the health care centres, which may further cause delay as small health facilities, including primary health centres and rural hospitals are not equipped with desired specialist manpower and blood transfusion facilities.

Nambiyal A [4] in her study, there were total 170 (1.98\%) cases of trivial to intractable postpartum hemorrhage during the study period, out of which there were 74 (0.9\%) cases of trivial PPH, 81 (1\%) of Severe PPH and 15 (0.2\%) of intractable PPH. The incidence of PPH was $1.98 \%$ of total deliveries. Out of 170 cases of PPH trivial PPH accounted for 43.53\%, severe PPH for 47.65\% and intractable PPH was 8.82\%. Cohen et al(1995)204 observed PPH in 2 to $10 \%$ of all deliveries, out of which severe PPH accounted for 1\% of the cases. Various authors, Sheikh et al., Bateman et al. and Lutomski et al. [5-7], estimated the incidence of postpartum hemorrhage as $1 \%$ to $5 \%$, which is comparable to our study and in another study by Rueangchainikhom et al. [8] the incidence of postpartum hemorrhage was $1.98 \%$.

In another study conducted in a tribal area of central India, by Bang et al. [9], the incidence of primary postpartum hemorrhage was 3.2\%. Sheikh et al. [5] reported the incidence of intractable PPH in $0.64 \%$ of the total deliveries, whereas in our study it was $0.2 \%$ of total deliveries. 
In the present study, the incidence of atonic PPH was less than $1 \%$. The low incidence could be due to adoption of AMTSL for third stage management in all cases. There were 54 cases (46.55\%) of mild PPH, who were successfully managed by medical methods. Uterine balloon tamponade was used in those cases, who did not adequately respond to medical management (31.89\%). Surgical intervention in the form of either B Lynch suture or stepwise devascularisation including internal iliac ligation was performed in 5 cases and obstetric hysterectomy was required in 20 cases. Availability of all modalities of treatment for PPH under one roof, expert opinions, surgical expertise, ICU with intensivist's back up, senior anaesthetists and availability of adequate blood and component therapy have contributed in saving all lives and preventing maternal mortality.

There is definite role of uterine balloon therapy (UBT) in management of atonic PPH. The device is easy to use, cheap and can be inserted by health worker like ANM or medical officer at primary health centres. There is enough evidence in support of usefulness of UBT in control or reduction of atonic PPH [10-16]. The UBT can either control or stop the blood loss or at least reduce the blood loss in severe cases of atonic PPH, while patient is being transferred to higher centre. Training of PHC staff in diagnosis of atonic PPH and differentiating between atonic and traumatic PPH is essential before introduction of UBT. Preparedness of tertiary care centre to deal with obstetric emergencies is very essential. Avoidance of any delay helps in reducing the blood loss. Intelligent anticipation regarding PPH, while delivering high risk woman helps in immediate interventions. Prompt communication at every level saves time and improves outcome. Communication from peripheral hospitals to a referral hospital before shifting the patient is helpful. That confirms the availability of specialist at the referral hospital and tertiary care centre gets time for it preparedness to deal with the emergency. Operation theatre and surgical team can be spared or prepared during transit time of the patient.

Encouraging institutional delivery has all the advantages as stated above. It reduces the need for transfer and saves time, limits the blood loss and keeps the patient condition stable till definite management is planned. High risk pregnancies are prone for development of PPH. Detection of high risk factors by peripheral health workers and timely transfer to higher centre can avoid the complication like PPH. Severe anaemia, severe hypertension, liver disorders and women with previous history of third stage complications must be refereed to higher centres for further evaluation. Delivery plan must be ensured for these women. The patient and relatives must be told about the risk of haemorrhage and risk to life of the woman.

Every primary health centre must keep its labour room ready for management of PPH. PPH tray must have all essential drugs especially the oxytocic drugs. A checklist of contents of PPH tray must be available in labour room. ANM and medical officers at PHC must monitor round the clock availability of essential drugs in PPH tray. Regular refresher training of PHC staff on common obstetric emergencies will help in improving their knowledge and skills required in saving lives. 


\section{Conclusion}

Atonic Postpartum haemorrhage is common among high risk pregnancies. Its incidence can be lowered by universal adoption of AMTSL. Institutional deliveries will help prevent and mange this complication in a effective manner. Facility Preparedness in all respect will prevent deaths due to atonic PPH. Wise and prompt decisions regarding use of different modalities or combination of modalities will save lives. Technical expertise, confidence and surgical skills are essential to mange complicated cases. Anaesthesia, ICU back up and availability of blood and component facilities improve clinical outcome. Avoidance of delays in identification and transfer, high risk pregnancy identification and timely referral, capacity building of peripheral health workers in use of medication and uterine balloon therapy will go a long way in reducing the maternal mortality related to atonic postpartum haemorrhage.

\section{References}

1. Dildy GA. Postpartum hemorrhage: New management options. Clin. Obstet Gyneco 45 (2002): 330-344.

2. Fenton JJ, Baumeister LM, Fogarty J. Active management of third stage of labour among American Indian women. Fam Med 37 (2005) :410-414.

3. Maughan KL, Heim, SW, Galazka SS. Preventing post-partum haemorrhage: managing the third stage of labour. AAFP 73 (2006): 1025-1028.

4. MUHS Nashik. A Prospective Study of Women Presenting With Trivial To Intractable Postpartum Hemorrhage With Special Reference to Comparison of Different Modalities Used For Prevention of Postpartum Hemorrhage. Published study MUHS Nashik (2014).

5. Shaikh S, Shaikh NB, Talpur S, et al. Postpartum hemorrhage: an experience at tertiary care hospital, Hyderabad. Quarterly Medical Channel 19 (2013).

6. Bateman BT, Berman MF, Riley LE, The epidemiology of postpartum hemorrhage in a large, nationwide sample of deliveries. Anesthesia and analgesia 110 (2010): 1368-1373.

7. Lutomski J, Byrne B, Devane D, et al. Increasing trends in atonic postpartum haemorrhage in Ireland: an 11-year population-based cohort study. BJOG: An International Journal of Obstetrics and Gynaecology 119 (2012): 306-314.

8. Rueangchainikhom W, Srisuwan S, Prommas S, et al. Risk factors for primary postpartum hemorrhage in Bhumibol Adulyadej Hospital. Journal of the Medical Association of Thailand. Chotmaihet thangphaet 92 (2009): 1586.

9. Bang RA, Bang AT, Reddy MH, et al. Maternal morbidity during labour and the puerperium in rural homes and the need for medical attention: A prospective observational study in Gadchiroli, India. BJOG: An International Journal of Obstetrics and Gynaecology 111 (2004): 231-228.

10. Tindell K, Garfinkel R, Abu-Haydar E, et al. Uterine balloon tamponade for the treatment of postpartum haemorrhage in resource-poor settings: a systematic review. BJOG 120 (2013): 5-14.

11. Burke TF, Ahn R, Nelson BD, et al. A postpartum haemorrhage package with condom uterine balloon tamponade: a prospective multi-centre case series in Kenya, Sierra Leone, Senegal, and Nepal. BJOG 123 (2016): 1532-1540. 
12. Pendleton AA, Natarajan A, Ahn R, et al. Emergency hysterectomy for uncontrolled postpartum hemorrhage may be averted through uterine balloon tamponade in Kenya and Senegal. Int J Gynecol Obstet 133 (2016): 124.

13. Pendleton AA, Natarajan A, Ahn R, et al. A qualitative assessment of the impact of a uterine balloon tamponade package on decisions regarding the role of emergency hysterectomy in women with uncontrolled postpartum hemorrhage in Kenya and Senegal. BMJ Open 6 (2016): e010083.

14. Herrick T, Mvundura M, Abu-Haydar E, et al. Impact modeling of a low cost uterine balloon tamponade package. Accepted, BMC Pregnancy and Childbirth (2017).

15. Mvundura M, Abu-Haydar E, Okoth E, et al. Cost effectiveness of the uterine balloon tamponade to control severe post-partum hemorrhage in Kenya.Int J Gynaecol Obstet 137 (2017): 185-191.

16. Burke TF, Danso-Bamfo S, Cappetta A, et al. An ultra-low-cost uterine balloon tamponade package saves lives among women with advanced shock from uncontrolled postpartum hemorrhage in low resource settings. IJGO (2017): 12251.

Citation: Vidyadhar Bangal. Incidence, Management and Outcome of Atonic Postpartum Haemorrhage at Tertiary Care Hospital. Obstetrics and Gynecology Research 1 (2018): 045-050. 